\title{
Perfil epidemiológico dos casos confirmados de infecção por COVID-19 no estado de Minas Gerais
}

\begin{abstract}
RESUMO | Objetivo: identificar o perfil epidemiológico dos casos confirmados de infecção por COVID-19 no Estado de Minas Gerais. Método: trata-se de um estudo descritivo, exploratório, retrospectivo, com abordagem quantitativa, realizada em um banco de dados público da Secretaria Estadual de Saúde de Minas Gerais. A amostra foi composta por 275 pacientes notificados durante o $1^{\circ}$ trimestre de 2020, os quais foram confirmados com COVID-19 em Minas Gerais. Resultados: observou-se prevalência de infecção na cidade de Belo Horizonte (59,3\%), seguido das cidades de Juiz de Fora (8,4\%) e Nova Lima (7,3\%). Com relação ao sexo e idade, foram acometidos em sua maioria adultos jovens com prevalência do sexo masculino $(59,6 \%)$ e idade entre 20 e 59 anos (80,7\%). Conclusão: o coronavírus apresentou alta transmissibilidade, bem como alta difusibilidade, em um curto espaço de tempo no território mineiro, sendo os adultos jovens os principais veículos carreadores para os idosos.

Palavras-Chave: Alnfecções por coronavírus; Epidemias; Prevenção de doenças; Controle de doenças transmissíveis; Saúde
\end{abstract} pública.

\begin{abstract}
Objective: to identify the epidemiological profile of confirmed cases of COVID-19 infection in the state of Minas Gerais. Method: this is a descriptive, exploratory, retrospective study, with a quantitative approach, carried out in a public database of the Minas Gerais State Department of Health. The sample was composed of 275 patients notified during the 1st quarter of 2020, who were confirmed with COVID-19 in Minas Gerais. Results: prevalence of infection was observed in the city of Belo Horizonte (59.3\%), followed by the cities of Juiz de Fora (8.4\%) and Nova Lima (7.3\%). Regarding sex and age, most young adults were affected, with a prevalence of males (59.6\%) and ages between 20 and 59 years (80.7\%). Conclusion: the coronavirus showed high transmissibility, as well as high diffusibility, in a short period of time in the territory of Minas Gerais, young adults being the main carriers for the elderly.
\end{abstract}

Keywords: Coronavírus infections; Epidemics; Disease prevention; Communicable disease control; Public health.

RESUMEN | Objetivo: identificar el perfil epidemiológico de casos confirmados de infección por COVID-19 en Minas Gerais. Método: se trata de un estudio descriptivo, exploratorio, retrospectivo, cuantitativo, realizado en una base de datos pública de la Secretaría de Salud del Estado de Minas Gerais. La muestra se compone de 275 pacientes notificados durante el primer trimestre de 2020, que fueron confirmados con COVID-19 en Minas Gerais. Resultados: la prevalencia de infección se observó en la ciudad de Belo Horizonte $(59,3 \%)$, seguida de Juiz de Fora $(8,4 \%)$ y Nova Lima $(7,3 \%)$. En cuanto al género y la edad, la mayoría de los adultos jóvenes estaban afectados, con una prevalencia de varones $(59,6 \%$ ) y con edades comprendidas entre los 20 y los 59 años $(80,7 \%)$. Conclusión: el coronavirus presentó una alta transmisibilidad y difusibilidad, en un corto espacio de tiempo en el territorio minero, siendo los adultos jóvenes los principales vehículos para los ancianos.

Palabras claves: Infecciones por coronavirus, epidemias, prevención de enfermedades, control de enfermedades transmisibles, salud pública.

\section{Patrick Leonardo Nogueira da Silva}

Enfermeiro, Mestrando pelo Programa de Pós-Graduação em Cuidado Primário em Saúde da Universidade Estadual de Montes Claros (PPGCPS/UNIMONTES). Montes Claros, MG, Brasil.

ORCID: 0000-0003-2399-9526

\section{Adélia Dayane Guimarães Fonseca Enfermeira, Doutora em Ciências da Saúde, Professora do Departamento de Enfermagem da Universidade Federal de Juiz de Fora (UFJF). Juiz de Fora, MG, Brasil. ORCID: 0000-0002-1168-7106}

\section{Edna de Freitas Gomes Ruas \\ Enfermeira, Mestre em Enfermagem, Professora do Departamento de}

Enfermagem da Universidade Estadual de Montes Claros (UNIMONTES). Montes Claros, Minas Gerais, Brasil.

ORCID: 0000-0002-4654-0817

\section{Carolina dos Reis Alves}

Enfermeira, Doutora em Ciências da Saúde, Professora do Departamento de Enfermagem da Faculdade Santo Agostinho (FASA). Montes Claros, MG, Brasil.

ORCID: 0000-0003-2107-6306

\section{Simone Guimarães Teixeira Souto \\ Enfermeira, Mestre em Enfermagem, Pro- fessora do Departamento de Enfermagem da Universidade Estadual de Montes Cla- ros (UNIMONTES). Montes Claros, MG, Brasil. \\ ORCID: 0000-0003-0562-005X}

\section{Cláudio Luís de Souza Santos}

Enfermeiro, Especialista em Saúde Mental pela Residência Multiprofissional da Universidade Estadual de Montes Claros (UNIMONTES). Montes Claros, MG, Brasil. ORCID: 0000-0002-9127-6349

Recebido em: 21/07/2021

Aprovado em: 23/07/2021 
INTRODUÇÃO

$\mathrm{O}$ COVID-19, cujo agente etiológico é o coronavírus, é uma doença infecciosa de alta transmissibilidade causada por um novo vírus que nunca havia sido identificado em seres humanos. O novo agente etiológico, no Brasil, foi descoberto em 2019 após casos registrados na China. As primeiras cepas virais em humanos foram isoladas pela primeira vez em 1937. No entanto, foi em 1965 que o vírus foi descrito como coronavírus em decorrência de sua morfologia microscópica associar-se a uma coroa ${ }^{1}$. A maioria das pessoas tem contato com as cepas virais mais comuns ao longo da vida, sendo as crianças pequenas mais propensas a se infectarem com o tipo mais comum. As espécies virais mais comuns que infectam humanos são o $\alpha$-coronavírus $229 \mathrm{E}$, $\alpha$-coronavírus NL63, $\beta$-coronavírus OC43 e $\beta$-coronavírus HKU12.

A sintomatologia viral da COVID-19 é decorrente de uma doença respiratória semelhante à gripe apresentando manifestações clínicas como tosse, febre e, em casos mais graves, pneumonia. É possível se proteger ao lavar as mãos com freqüência e evitar tocar no rosto. A principal forma de contaminação pelo COVID-19 se dá por meio do contato com uma pessoa acometida, de modo a transmitir o vírus por meio de tosse, espirros, gotículas de saliva ou coriza ${ }^{3}$.

As medidas profiláticas contra a aquisição da COVID-19 compreendem: lavagem freqüente das mãos com água e sabonete por pelo menos 20 segundos ou desinfetante alcoólico para as mãos na ausência de água e sabonete; evitar tocar nos olhos, nariz e boca com as mãos não lavadas; evitar contato direto com pessoas doentes; cobrir boca e nariz ao tossir ou espirar com um lenço de papel e jogá-lo no lixo; e limpar e desinfetar objetos e superfícies tocados com freqüência. Em caso de suspeita da doença, indica-se o repouso e hidratação oral freqüente, além de medidas para o alívio sintomático, tal como o uso de analgésicos e antitérmicos ${ }^{4}$.

Em situações mais graves, onde ocorrem sintomas mais graves como uma febre alta e persistente, bem como dificuldade respiratória (dispnéia), faz-se importante a busca pela Unidade Básica de Saúde (UBS) tendo em vista a notificação do caso suspeito e o monitoramento da doença de modo a objetivar a sua confirmação pelo COVID-19 ou o seu pleno descarte diagnóstico ${ }^{5}$. Diante de caso suspeito, o serviço de saúde deverá fornecer máscara cirúrgica para o paciente e conduzi-lo para sala isolada. Já os profissionais de saúde devem utilizar equipamento de proteção individual (EPI) que, com exceção da proteção ocular, deverá ser descartado após utilização ${ }^{6}$.

De acordo o Ministério da Saúde (MS), até o presente momento, já foram notificados no Brasil 6.836 casos confirmados de infecção por COVID-19 e 241 mortes, sendo a taxa de letalidade de $3,5 \%{ }^{7}$. Entre mais de 200 mortes analisadas, $89 \%$ foram de pessoas com mais de 60 anos e $84 \%$ de pessoas com pelo menos uma comorbidade, que é uma doença pré-existente. Sete das 212 mortes analisadas ocorreram em pessoas com menos de 60 anos e sem comorbidades $^{8}$. O histórico de viagem de pacientes para áreas com transmissão do COVID-19, contatos com pessoas de caso suspeito ou confirmado da doença, são fatores que os profissionais de saúde devem levar em consideração

\begin{tabular}{|c|c|c|}
\hline Variáveis & n & $\%$ \\
\hline Casos suspeitos & 34.224 & 100,0 \\
\hline Casos confirmados & 275 & 0,80 \\
\hline Óbitos em investigação & 40 & 0,11 \\
\hline Óbitos confirmados & 02 & 0,0058 \\
\hline
\end{tabular}

Fonte: COES MINAS/COVID-19/SESMG. Atualizado em 31/03/2020. para notificar as Secretarias Municipais de Saúde que, por sua vez, acionarão a Secretaria de Estado de Saúde de Minas Gerais (SESMG) e o MS ${ }^{6}$.

No intuito de impedir a disseminação do novo COVID-19, o Núcleo de Vigilância Epidemiológica, Ambiental e de Saúde do Trabalhador da Regional de Saúde de Montes Claros alertou os profissionais de saúde quanto à importância do reforço das ações de vigilância e assistência em saúde, coleta de amostras para a realização de exames laboratoriais e implementação de ações de comunicação, de modo a objetivar o esclarecimento da população sobre as medidas preventivas que devem ser adotadas para evitar a infecção pelo vírus ${ }^{6}$.

Sendo assim, objetivou-se identificar o perfil epidemiológico dos casos confirmados de infecção por COVID-19 no Estado de Minas Gerais, Brasil.

\section{MÉTODOS}

Trata-se de um estudo com delineamento descritivo, exploratório, retrospectivo, com abordagem quantitativa, realizado no banco de dados público da SESMG. A distribuição dos casos suspeitos por município, bem como dos casos descartados e notificados, são atualizados diariamente, e podem ser visualizados publicamente e baixados por meio do site http://www. saude.mg.gov.br/coronavirus, no link "Distribuição dos casos de COVID-19 em Minas Gerais". Até o momento, estão totalizados 34.224 casos suspeitos 
para COVID-19 e 275 casos confirmados. Quarenta óbitos estão em investigação e dois óbitos foram confirmados. A amostra deste estudo foi composta por 275 pacientes notificados com confirmação de infecção. O período das notificações se deu durante os meses de janeiro, fevereiro e março de 2020 (Tabela 1).

Foi adotado o seguinte critério de inclusão para participação da pesquisa: casos de COVID-19 devidamente notificados e confirmados durante o $1^{\circ}$ trimestre de 2020 no Estado de Minas Gerais. Até o momento, foram notificados 40 óbitos suspeitos, sendo 20 descartados para COVID-19. Os demais óbitos suspeitos aguardam a realização de exames laboratoriais e levantamento de informações clínicas e epidemiológicas. Considerando que o MS classificou todo o país como transmissão comunitária, além da necessidade de qualificar as informações sobre a circulação do COVID-19 em Minas Gerais, a partir de 23/03/2020 o Boletim Informativo Diário COVID-19 publicou o detalhamento apenas dos casos confirmados.

Foi utilizado um formulário semiestruturado como instrumento de coleta de dados. Os dados foram tabulados pelo Programa Statistical Package for the Social Sciences (SPSS), representados por meio de tabelas e gráficos construídos através do Microsoft Excel®, versão 2007, e disponibilizados pela SESMG. As variáveis quantitativas foram expostas por meio de freqüências absolutas (n) e percentuais (\%). A análise dos dados se deu por meio de epidemiologia descritiva simples não-paramétrica e não probabilística. O estudo obedeceu aos preceitos éticos estabelecidos pela Resolução $n^{\circ} 466$, de 12 de dezembro de 2012, do Conselho Nacional de Saúde (CNS), a qual regulamenta a realização de pesquisa envolvendo seres humanos ${ }^{9}$. Por se tratar de dados de domínio público, não houve a necessidade de encaminhar a pesquisa para apreciação e aprovação do Comitê de Ética em Pesquisa (CEP).

\section{RESULTADOS}

Perfil dos casos suspeitos e óbitos confirmados

Os óbitos em investigação constituem-se casos suspeitos os quais aguardam a realização de exames laboratoriais e levantamento de informações clínicas e epidemiológicas. Até o momento foram notificados 61 óbitos suspeitos, sendo 19 descartados para COVID-19.

O primeiro paciente que veio a óbito é do sexo feminino, 82 anos, residente no município de Belo Horizonte. Foi internada no Hospital Biocor, em Nova Lima, em 21/03/2020, com quadro de febre, tosse e desconforto respiratório. Transferida para UTI em $23 / 03 / 20$. Foi coletado swab de secreção orofaríngea para pesquisa de COVID-19 em 23/03/2020. A mesma apresentava-se com as seguintes comorbidades: doença cardiovascular (DCV) crônica, Diabetes Mellitus (DM) e pneumopatia crônica. O óbito ocorreu em 29/03/2020. O exame de swab detectável para COVID-19 foi realizado em laboratório privado.

Com relação ao segundo paciente, sexo masculino, 66 anos, residente do município de Belo Horizonte, portador de cardiopatia e DM. O exame foi detectável por meio de reação da transcriptase reversa seguida de reação em cadeia de polimerase (RT-PCR) em laboratório da rede privada. Foi coletada outra amostra orofaríngea para realização de swab na Fundação Ezequiel Dias (FUNED). O óbito ocorreu em 30/03/2020.

\section{Perfil dos casos confirmados}

Foi observada a confirmação da doença em 32 cidades mineiras durante o $1^{\circ}$ trimestre de 2020. Com isso, as notificações foram menos freqüentes em janeiro e fevereiro e mais freqüentes durante todo o mês de março, bem como suas confirmações. Sendo assim, a COVID-19 foi majoritariamente prevalente na cidade de Belo Horizonte (59,3\%), no Estado de Minas Gerais, seguido de Juiz de Fora $(8,4 \%)$ e Nova Lima (7,3\%) (Tabela 2).

Enfatiza-se a existência de cinco casos que ainda estão sob investigação e aguardando os resultados de exames para a sua confirmação ou descarte (Tabela 2). Este agente etiológico apresentou uma maior manifestação quanto à sua confirmação via exames de sorologia laboratorial em pacientes do sexo masculino $(59,6 \%)$, sendo estes adultos jovens com idade entre 20 e 59 anos $(80,7 \%)$ (Tabela 3).

Em Minas Gerais, durante os meses de janeiro e fevereiro, as notificações não foram tão incidentes. A partir do mês de março que estas notificações passaram a ser mais efetivas. Da Semana Epidemiológica (SE) $n^{\circ} 10$ para a SE $n^{\circ} 11$, houve uma variação de 451 notificações em todo o território mineiro. Na SE $n^{\circ} 12$, essa diferença foi 12 vezes maior quando comparada com a semana anterior, sendo de 7.371 notificações. Já na última SE deste mês, sendo esta a de $n^{\circ} 13$, as notificações atingiram um auge três vezes maior do que na semana anterior, somando um quantitativo de 12.341 novos casos para serem investigados (Figura 1).

\section{DISCUSSÃO}

O COVID-19, no Brasil e no mundo, configura-se uma ameaça totalmente desconhecida e ainda não estudada. Não se sabe ao certo todas as conseqüências fisiopatológicas, bem como a gravidade destas, que este antígeno é capaz de estabelecer após sua infecção no organismo humano. O vírus é mais facilmente difundido conforme o aglomeramento geoespacial de pessoas em um mesmo ambiente. Grandes 


\begin{tabular}{|c|c|c|c|c|c|}
\hline Município de residência* & $\mathbf{n}$ & $\%$ & $\begin{array}{l}\text { Município de } \\
\text { residência* }\end{array}$ & $\mathbf{n}$ & $\%$ \\
\hline Belo Horizonte & 163 & 59,3 & Bom Despacho & 01 & 0,4 \\
\hline Juiz de Fora & 23 & 8,4 & Campo Belo & 01 & 0,4 \\
\hline Nova Lima & 20 & 7,3 & Campos Altos & 01 & 0,4 \\
\hline Uberlândia & 10 & 3,6 & $\begin{array}{l}\text { Carmo do } \\
\text { Cajuru** }^{*}\end{array}$ & 01 & 0,4 \\
\hline Divinópolis & 09 & 3,3 & $\begin{array}{c}\text { Coronel } \\
\text { Fabriciano }\end{array}$ & 01 & 0,4 \\
\hline Betim & 05 & 1,8 & Extrema & 01 & 0,4 \\
\hline Contagem & 05 & 1,8 & Guimarânia*** & 01 & 0,4 \\
\hline Sabará & 03 & 1,1 & Ipatinga & 01 & 0,4 \\
\hline Uberaba & 03 & 1,1 & Manhuaçu**** & 01 & 0,4 \\
\hline Governador Valadares & 02 & 0,7 & Muriaé & 01 & 0,4 \\
\hline Lagoa da Prata & 02 & 0,7 & Patrocínio & 01 & 0,4 \\
\hline Mariana & 02 & 0,7 & Santa Luzia & 01 & 0,4 \\
\hline Poços de Caldas & 02 & 0,7 & Serra do Salitre & 01 & 0,4 \\
\hline São João del Rei & 02 & 0,7 & Timóteo & 01 & 0,4 \\
\hline Sete Lagoas & 02 & 0,7 & Unaí & 01 & 0,4 \\
\hline Alfenas & 01 & 0,4 & Em investigação & 05 & 1,8 \\
\hline Boa Esperança & 01 & 0,4 & & & \\
\hline
\end{tabular}

\section{Fonte: COES MINAS/COVID-19/SESMG.}

*Dados atualizados diariamente pelas Unidades Regionais de Saúde.

**Caso inicialmente notificado como residente em Divinópolis.

***Caso inicialmente notificado como residente em Patos de Minas.

$* * * *$ Caso inicialmente notificado como residente em Curvelo. Atualizado em 31/03/2020.

regiões metropolitanas contribuem para esta rápida circulação viral tendo em vista o alto índice populacional de habitantes, bem como o alto índice de visitantes ${ }^{10}$. A cidade de Belo Horizonte, capital do Estado de Minas Gerais, localizada na região sudeste do Brasil, apresenta uma população estimada de 2.512.070 habitantes ${ }^{11}$. Ainda, é referência de educação de qualidade sendo pólo de múltiplas faculdades. Apresenta pontos turísticos que são atrativos para viajantes. Sendo assim, estes fatores contribuem para uma disseminação viral mais rápida e conseqüente manifestação sintomática e possível agravamento clínico em pessoas que compõe o grupo de vulnerabilidade. Neste estudo foi confirmada a infecção em 32 cidades mineiras de janeiro a março de 2020. Sendo assim, a COVID-19 foi predominantemente prevalente na capital mineira, bem como em cidades circunvizinhas, decorrente do fluxo demográfico.

Em Minas Gerais, a vulnerabilidade mostrou maior prevalência em pessoas do sexo masculino $(59,6 \%)$ quando comparadas ao sexo feminino $(40,4 \%)$. Por meio de uma pesquisadora do Departamento de microbiologia molecular e imunologia da Escola de Saúde Pública da Universidade Johns Hopkins, nos EUA, é abordado que o estrogênio - hormônio sexual feminino - pode ser a explicação para as diferentes respostas ao COVID-19. O estrogênio pode estimular aspectos da imunidade que são importantes para eliminar uma infecção viral e responder bem às vacinas. Com base nessas informações, acredita-se que o estrogênio possa ser um fator contributivo para a maior imunidade do sexo feminino no surto atual de coronavírus, porém, tendo em vista que o surto é recente, ainda não há pesquisas efetivas que demonstrem isso de forma definitiva(12).

O COVID-19 mostrou ser mais prevalente em pacientes adultos jovens, com idade compreendida entre 20 e 


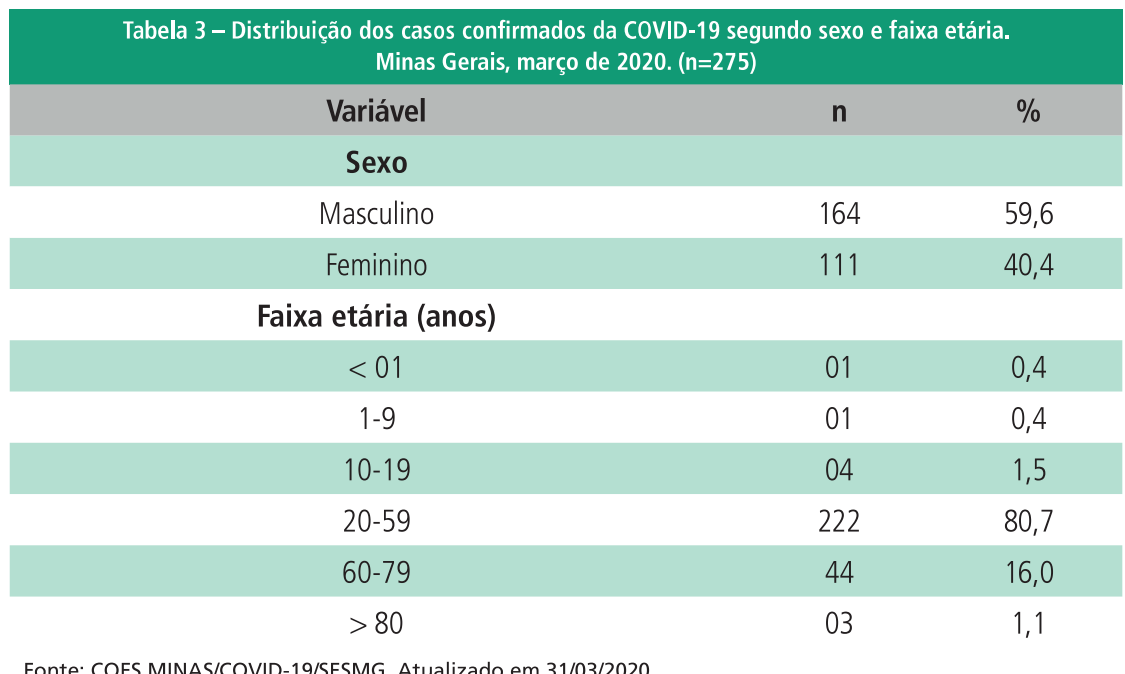

Fonte: COES MINAS/COVID-19/SESMG. Atualizado em 31/03/2020

Figura 1 - Número de casos notificados de COVID-19, segundo semana epidemiológica e data de notificação. Minas Gerais, março de 2020.

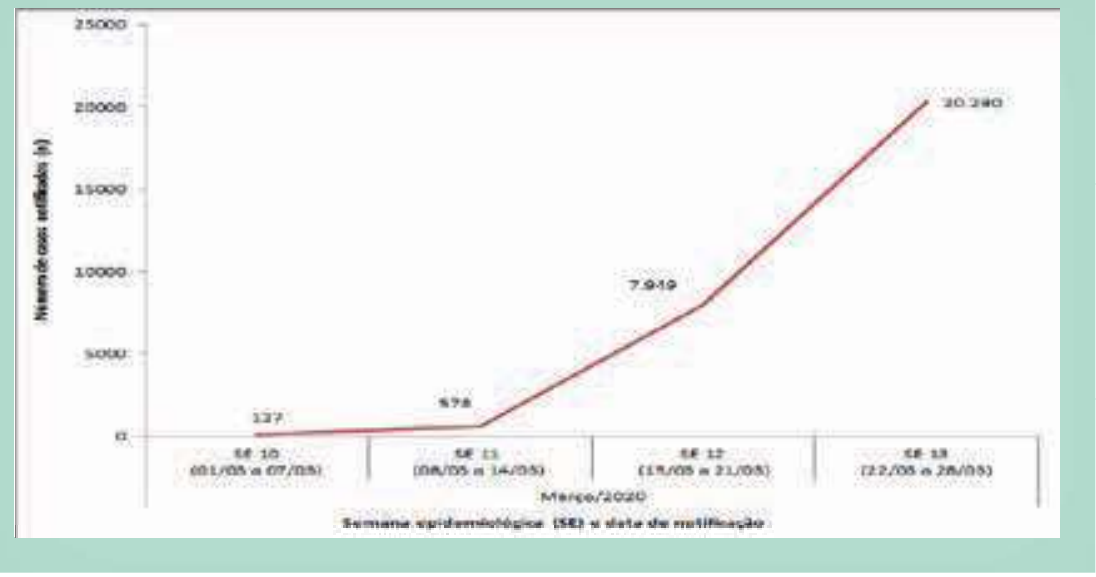

Fonte: FormSUSRedCap e e-SUS VE/COES MINAS/COVID-19/SESMG. Atualizado em 31/03/2020.

59 anos $(80,7 \%)$. Este dado sugere que pessoas mais jovens podem apresentar maior capacidade de veiculação, o que pode facilitar a transmissibilidade viral. A doença está vinculada a grande impacto socioeconômico em escala mundial, sendo mais grave nos idosos em decorrência da baixa imunidade e resistência física, além de comorbidades associadas. Quando comparados a um individuo jovem, encontram-se no grupo de risco e estão mais susceptíveis às conseqüências mais graves deste vírus, tal como o óbito(13). O COVID-19 em crianças e adolescentes é responsável internados em Montes Claros apresentando-se hemodinamicamente estáveis, um foi admitido no Centro de Terapia Intensiva (CTI), 34 aguardam resultado de exame confirmatório, 328 em investigação e quatro pacientes de Montes Claros internados em outros municípios. Ainda não houve óbitos notificados e nem confirmados por COVID-19 na região norte de Minas Gerais ${ }^{15}$. Em Espinosa, outro município norte mineiro, também foi notificado 17 pacientes sintomáticos e com suspeita, um óbito suspeito e nenhum caso confirmado ou descartado ${ }^{16}$.

Esta doença tomou dimensões pandêmicas com repercussões caóticas. Números expressivos de adoecimentos e óbitos em um curto espaço de tempo, impacto direto na economia mundial e insuficiência de leitos em Unidade de Terapia Intensiva (UTI) dos hospitais para internação nos casos sintomáticos mais graves da doença ${ }^{17}$. Tendo em vista a atual conjuntura do Brasil e do mundo, foi adotado como principal medida profilática o isolamento social de modo a evitar o contato direto e, conseqüentemente, retardar a transmissão viral, bem como os óbitos, fazendo-se com que as instituições de saúde suportem a demanda de pacientes acometidos em longo prazo até que seja instaurado um tratamento eficaz contra essa doença e que supra de maneira satisfatória toda a demanda emergencial ${ }^{18,19,20}$.

\section{CONCLUSÃO} tes com surtos, sendo geralmente mais leve que os adultos, mas pode progredir para doença grave em alguns casos. Até os recém-nascidos ( $R N$ ) podem sofrer do COVID-19 e as crianças podem desempenhar um papel de disseminador na comunidade ${ }^{14}$.

Até o mês de março de 2020, o município de Montes Claros, macrorregião do norte de Minas Gerais, apresentava 389 pacientes suspeitos notificados com sintomatologia clínica sugestiva. Destes, 60 foram descartados para a doença, quatro foram
Por meio deste estudo, foi possível identificar o perfil epidemiológico dos casos notificados e confirmados de infecção por COVID-19 no Estado de Minas Gerais, Brasil, durante o $1^{\circ}$ trimestre de 2020. O Brasil, no seu atual panorama, vive uma crise na saúde por meio da pandemia do COVID-19. A economia é afetada, pois o vírus se dissemina rapidamente de uma forma que o setor da saúde não consegue administrar eficazmente tendo em vista 
a alta demanda e o alto fluxo de pacientes contaminados pela doença. Foi preciso adotar, em caráter emergencial, o isolamento social a fim de retardar a transmissibilidade viral e a demanda destes pacientes no hospital, de modo a contribuir com a redução de óbitos por esta afecção.

Portanto, o COVID-19 apresentou alta transmissibilidade, bem como alta difusibilidade, em um curto espaço de tempo no território mineiro. A maior concentração de vítimas infectadas se deu na Capital mineira, sendo esta Belo Horizonte, e cidades circunvizinhas. Neste estudo, houve a confirmação de dois óbitos de idosos por COVID-19. Isso mostra que os idosos configuram o principal grupo de risco para o acometimento desta doença, bem como para as complicações graves e óbito. Pode-se inferir que os adultos jovens deste estudo são os veículos carreadores para o público-alvo mais susceptível que são os idosos.

Este estudo contou com a discussão de dados inéditos, porém parciais. As notificações e confirmações continuarão a ser feitas de forma contínua pelos municípios e Estados brasileiros de forma a alimentar os sistemas de informação do MS diariamente confor- me a confirmação do diagnóstico. O primeiro caso de COVID-19 foi descoberto em dezembro de 2019, porém as primeiras notificações foram realizadas a partir de fevereiro de 2020. Sendo assim, o estudo se limitou ao banco de dados das notificações confirmadas pela doença em Minas Gerais apenas dos três primeiros meses de 2020. Conforme as confirmações forem sendo divulgadas posteriormente, sugere-se a complementação de novos estudos comparativos a respeito da progressão da doença.

\section{Referências}

1. Silva LJ. A globalização da doença. Rev. Saúde Pública [Internet]. 2003 [cited $2020 \mathrm{abr}$ 3];37(3):273-4. doi: https://doi.org/10.1590/S003489102003000300001

2. Nobre AFS, Sousa RCM, Santos MC, Barbagelata LS, Costa Júnior E, Lima DF, et al. Primeira detecção de coronavírus humano associado à infecção respiratória aguda na Região Norte do Brasil. Rev. Pan-Am. Saúde [Internet]. 2014 [cited 2020 abr 3];5(2):37-41. doi: http://dx.doi.org/10.5123/S217662232014000200005

3. Brasil. Ministério da Saúde. Secretaria de Atenção Primária à Saúde. Protocolo de manejo clínico do coronavírus (COVID-19) na atenção primária à saúde [Internet]. Brasília: MS, 2020 [cited 2020 abr 3]. Available from: https://www. unasus.gov.br/especial/covid19/pdf/37

4. Brasil. Ministério da Saúde. Secretaria de Atenção Primária à Saúde. Manejo de corpos no contexto do novo coronavírus COVID-19 [Internet]. Brasília: MS, 2020 [cited 2020 abr 3]. Available from: https://www.saude.gov.br/images/ $\mathrm{pdf} / 2020 / \mathrm{marco} / 25 / \mathrm{manejo}$-corpos-coronavirus-versa01-25mar20-rev5.pdf

5. Agência Nacional de Vigilância Sanitária. Orientações para serviços de saúde: medidas de prevenção e controle que devem ser adotadas durante a assistência aos casos suspeitos ou confirmados de infecção pelo novo coronavírus (SARS-CoV-2) [Internet]. Brasília: ANVISA, 2020 [cited 2020 abr 4]. Available from: https://www.unasus.gov.br/especial/covid19/pdf/23

6. Costa P. Seminário da Regional de Saúde de Montes Claros reforça a mobilização dos municípios contra Coronavírus, H1N1 e Sarampo [Internet]. Belo horizonte: SESMG, 2020 [cited 2020 abr 4]. Available from: https://www.saude. mg.gov.br/component/gmg/story/12209-seminario-da-regional-de-saude-de-montes-claros-reforca-a-mobilizacao-dos-municipios-contra-coronavirus-h1n1-e-sarampo

7. Valente J. Número de casos de COVID-19 no Brasil chega a 6.863 e mortes somam 241 [Internet]. Brasília: Agência Brasil, 2020 [cited 2020 abr 4]. Available from: https://agenciabrasil.ebc.com.br/saude/noticia/2020-04/ao-vivo-numero-de-casos-de-covid-19-no-Brasil-chega-6836-mortes-somam-241

8. Rio de Janeiro. Brasil tem 241 mortes e 6.836 casos confirmados de coronavírus; ministro diz que número é maior [Internet]. Rio de Janeiro: Jornal da Globo G1, 2020 [cited $2020 \mathrm{abr}$ 4]. Available from: https://g1.globo.com/bemestar/ coronavirus/noticia/2020/04/01/brasil-tem-240-mortes-e-6836-casos-confirmados-de-coronavirus-diz-ministerio.ghtml

9. Brasil. Ministério da Saúde. Conselho Nacional de Saúde. Resolução $n^{\circ} 466$, de 12 de dezembro de 2012 [Internet]. Regulamenta a realização de pesquisa envolvendo seres humanos. Brasília: CNS, 2012 [cited 2020 abr 4]. Available from: https://bvsms.saude.gov.br/bvs/saudelegis/cns/2013/res0466_12_12_2012.htm|
10. Brasil. Ministério da Saúde. Secretaria de Vigilância em Saúde. Centro de Operações de Emergências em Saúde Pública. Infecção humana pelo novo coronavírus (2019-nCoV). Bol. Epidemiol. [Internet]. 2020 [cited 2020 abr 7];1(1):117. Available from: https://www.saude.gov.br/images/pdf/2020/fevereiro/04/ Boletim-epidemiologico-SVS-04fev20.pdf

11. Instituto Brasileiro de Geografia e Estatística. Censo demográfico 2019 Belo Horizonte [Internet]. Rio de Janeiro: IBGE, 2019 [cited 2020 abr 5]. Available from: https://www.ibge.gov.br/cidades-e-estados/mg/belo-horizonte.html

12. Orgaz CJ. Coronavírus: por que há mais homens que mulheres infectados [Internet]. São Paulo: British Broadcasting Corporation News Brasil, 2020 [cited 2020 abr 7]. Available from: https://www.bbc.com/portuguese/internacional-51674894

13. Correia MITD, Ramos RF, Bahten LCV. Os cirurgiões e a pandemia do COVID-19. Rev Col. Bras. Cir. [Internet]. 2020 [cited 2020 abr 7]:47:e20202536. doi: https://doi.org/10.1590/0100-6991e-20202536

14. Choi S, Kim HW, Kang J, Kim DH, Cho EY. Epidemiology and clinical features of coronavirus disease 2019 in children. Clin. Exp. Pediatr. [Internet]. 2020 [cited 2021 jun 2];63(4):125-32. doi: https://doi.org/10.3345/cep.2020.00535

15. Minas Gerais. Secretaria Municipal de Saúde de Montes Claros. Coronavírus em Montes Claros - boletim epidemiológico. Montes Claros: SMS, 2020.

16. Minas Gerais. Secretaria Municipal de Saúde de Espinosa. Boletim epidemiológico $n^{\circ}$ 10/2020. Espinosa: SMS, 2020.

17. Vilela DAM. The value of mitigating epidemic peaks of COVID-19 for more effective public health responses. Rev. Soc. Bras. Med. Trop. [Internet]. 2020 [cited 2020 abr 7];53:e20200135. doi: http://doi.org/10.1590/0037-8682-01352020

18. Brasil. Ministério da Saúde. Secretaria de Vigilância em Saúde. Centro de Operações de Emergências em Saúde Pública. Especial: doença pelo coronavírus 2019. Bol. Epidemiol. [Internet]. 2020 [cited 2020 abr 7];esp(7):1-28. Available from: https://www.saude.gov.br/images/pdf/2020/April/06/2020-04-06-BE7-Boletim-Especial-do-COE-Atualizacao-da-Avaliacao-de-Risco.pdf

19. Gonçalves ADC, Binda ALM, Pinto EN, Oliveira ES, Binda Netto I. A mudança de decúbito na prevenção de lesão por pressão em pacientes na terapia intensiva. Rev. Nursing (São Paulo) [Internet]. 2020 [cited 2021 jul 21];23(265):4151 70. doi: http://doi.org/10.36489/nursing.2020v23i265p4151-4170

20. Luz DCRP, Campos JRE, Bezerra POS, Campos JBR, Nascimento AMV, Barros AB. Burnout e saúde mental em tempos de pandemia de COVID -19: revisão sistemática com metanálise. Rev. Nursing (São Paulo) [Internet]. 2021 [cited 2021 jul 21];24(276):5714-25. doi: http://doi.org/10.36489/nursing. 2021v24i276p5714-5725 